\title{
Thunderstorm Electrification
}

\author{
Stephanie Bradshaw ${ }^{1}$ \\ Department of Physics and Astronomy \\ Carthage College \\ Kenosha, WI
}

\begin{abstract}
Understanding how thunderstorms work is important as it can help assess risks associated with electrical activity in thunderstorms and other phenomena of a somewhat similar nature such as volcanic eruptions, heavy snowstorms, and large hurricanes. We started with a review of the scientific literature in order to grasp the current knowledge and understanding of electrification processes in thunderstorms. We then investigated what types of observations could be made using CAERENet (Carthage Atmospheric Electricity Research and Education Network) electric field mills. We also built mathematical models of electric charge, current, and electric field using Python. A comparison between computer model/simulations and electric field mill observed data would be used to better understand electrification processes in naturally occurring thunderstorms.
\end{abstract}

\section{Background}

Thunderstorms have been studied in the past in various approaches including balloon experiments. Balloons have been deployed into thunderstorms with an attached payload, which often included tools used to measure various parameters such as electric field. Electric field data suggested the existence of distinct charge regions inside of a thunderstorm due to the positive and negative electric field trends (Stolzenburg 2008). These regions are typically tripole structures. A normal polarity tripole structure has a lower region of positive charge, a middle negative charge region, and an upper positive charge region. Inverted polarity storms have charge regions opposite those in normal polarity storms (Rust 2005). They contain a lower negative charge region, a middle positive charge region, and an upper negative charge region, and the conditions in which inverted polarity storms form are not well-understood.

Both normal and inverted polarity structures are further complicated by screening charges and by variability of meteorological conditions within a thunderstorm. Screening charges typically form around the borders of the thunderstorm as a natural consequence of the conductivity of the atmosphere. Inside of a thunderstorm updraft and non-updraft regions can exist. Updraft regions experience vertical velocities exceeding one meter per second. Non-updraft regions experience downward vertical motion or heavy precipitation. The updraft and non-updraft regions inside of a thunderstorm tend to produce varied charge structures. Figure 1 shows the difference between the charge regions in an updraft versus a non-updraft region as adapted from Stolzenburg (2008). The updraft regions tend to have a normal tripole structure while the non-updraft regions tend to form a more complicated structure with extra charge regions.

${ }^{1}$ Funding provided by the Undergraduate Research Award through the Wisconsin Space Grant Consortium Advisor: Dr. Brant Carlson 


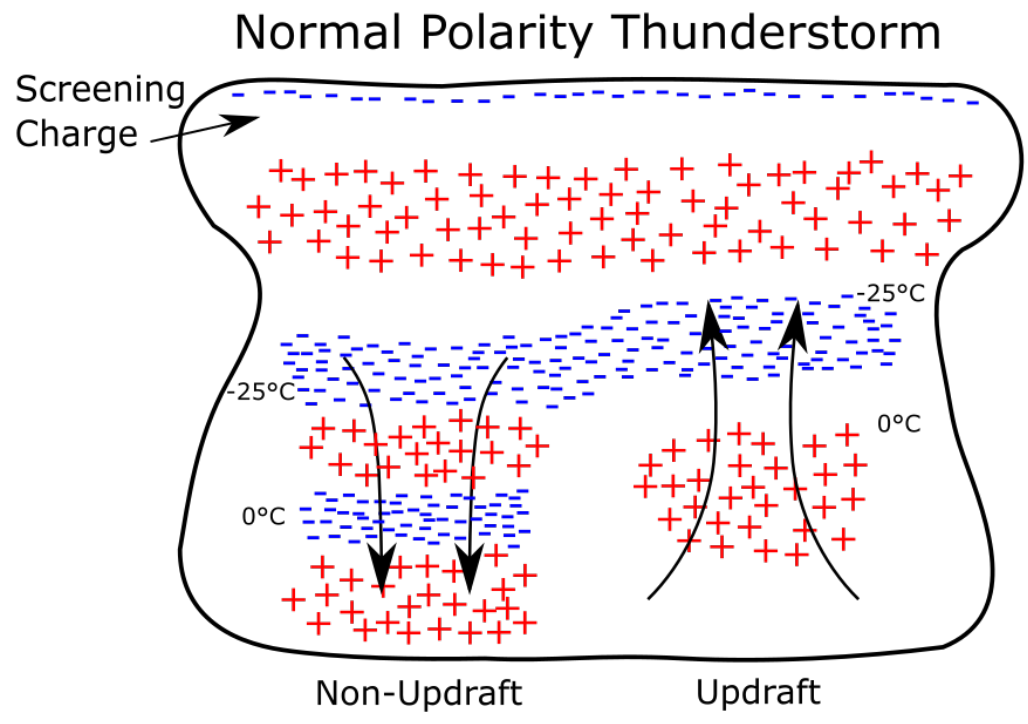

Figure 1: A representation of the positive (red) and negative (blue) charge regions of a thunderstorm with respect to an updraft and non-updraft structure. This model is adapted from Stolzenburg and Marshall's data (2008).

Throughout scientific history, there have been many theories explaining how thunderstorms electrify or how the charges are separated into the observed charge regions. Investigating literature sources revealed the dominance of one theory called the Relative Diffusional Growth Rate Theory (Saunders 2008). The theory involves the collision and separation of ice and graupel particles to form charge regions inside of a thunderstorm. Graupel is considered a soft hail and is formed when super-cooled water droplets rime a snowflake (NOAA 2010). Graupel is also a larger particle than an ice particle.

The Relative Diffusional Growth Rate Theory indicates charge is transferred between particles when they collide (Saunders 2008). The charge transferred depends on the growth rate of the ice and graupel particles. Whichever particle is growing fastest by vapour diffusion at collision will charge positively upon separation. This happens because the particle growing fastest at collision loses mass, while the particle growing slowest gains mass (Saunders 2008). The mass lost and gained is associated with negative charge because of the way ions diffuse in the particle (Dash 2001). Therefore, a neutrally charged particle that loses mass during a collision will become positively charge because there are less negative charges than positive charges. After charging, the particles move (by gravity and convection) to form regions of charge. Since graupel is a larger, it is more likely to fall down with gravity while the ice particle is more likely to move upwards with convection in a thunderstorm. Figure 2 shows a collision between a graupel and an ice particle and the resulting charge. Figure 3 shows how the Relative Diffusional Growth Rate Theory could work within a normal polarity tripole structure thunderstorm. 


\begin{tabular}{|l|l|l|}
\hline Before & Collision & After \\
\hline & & \\
\hline
\end{tabular}

Figure 2: A depiction of the Relative Diffusional Growth Rate Theory as a graupel particle (large particle) collides with an ice particle (small particle). The particles have an inner positive charge region (red) and outer negative charge region (blue) due to the way ions within particles (Dash 2001). The amount of color in each particle is not drawn to scale. Each particle starts out neutrally charged in the before section. The top row represents the graupel particle as growing faster than the ice particle. The bottom row represents the ice particle growing faster than the graupel particle. The up and down arrows indicate the direction of particle motion with gravity and convection.

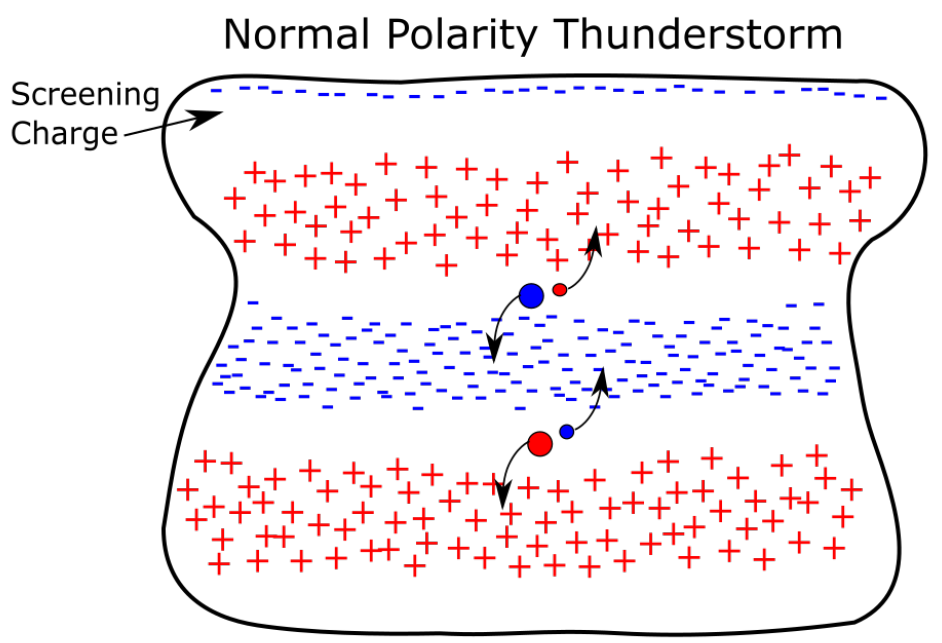

Figure 3: A representation of the Relative Diffusional Growth Rate Theory in a normal polarity thunderstorm. Red represents positive and blue represents negative charge values. Graupel particles are the larger circles while the smaller circles represent ice particles. The particles move upwards and downwards with gravity and convection.

The Relative Diffusional Growth Rate Theory has primarily been studied in laboratory settings such as in a cloud chamber (Saunders 2008). Cloud chambers have allowed scientists to study thunderstorms in a controlled, easily accessible environment. The problem with cloud chamber experiments is the conditions inside a thunderstorm tend to be difficult to attain in a cloud chamber, so extrapolating from cloud chamber results to thunderstorm conditions is difficult. 


\section{Methods}

Knowing what was found about thunderstorms from the literature, it is clear there are areas within thunderstorm electrification that are not well understood or observed. The Relative Growth Rate Theory has evidence supporting it within cloud chambers but few observations have been performed in a natural thunderstorm environment. We hope to use CAERENet electric field mills (an instrument which measures the electric field from the ground) to study the electrification processes in a natural environment by determining charge geometry and dynamics. In order to understand what kind of insight an electric field mill could lend to studying electrification processes, one needs to understand how to interpret electric field mill data to infer the charge geometry and dynamics of thunderstorms. Models were created using Python for the purpose of understanding how to interpret the electric field mill data.

The models utilized various mathematical methods including the Method of Images, the Poisson Equation, and the Steady State Charge Conservation Equations. The Poisson equation is $\nabla^{2} \varphi+\frac{\rho}{\epsilon_{0}}=0$ where $\varphi$ is the electric potential and $\rho$ is the charge density. The Steady State Charge Conservation Equations are $\nabla^{\prime} \cdot \dot{J}+\frac{\partial \rho}{\partial t}=0$ and $\dot{\nabla} \cdot\left(\sigma E+f_{s r c}\right)=0$ where $\dot{J}$ is the current, $\rho$ is the charge density, $J_{s r c}$ is the current at the source, $E$ is the electric field, and $\sigma$ is the conductivity. The Steady State Charge Conservation Equations can also be represented by the equation $\dot{\nabla} \sigma \cdot \dot{\nabla} \varphi+\sigma \nabla^{2} \varphi-\dot{\nabla} J_{s r c}=0$ with the variables being the same as in the first two Steady State Charge Conservation Equations. The equation for the Method of Images is $E_{y}=\frac{-2 Q h}{4 \pi \epsilon_{0}\left(a^{2}+h^{2}\right)^{\frac{3}{2}}}$ where $E_{y}$ is the electric field, $Q$ is the charge, $h$ is the height above the ground, and $a$ is the distance from the center of the storm to the electric field mill.

To model a thunderstorm, current regions (arrays of current values) and conductivity values were used as input values. Using these current and conductivity values, we used the Steady State Conservation Equation $\left(\dot{\nabla} \sigma \cdot \dot{\nabla} \varphi+\sigma \nabla^{2} \varphi-\dot{\nabla} J_{s r c}=0\right)$ to determine the electric potentials with boundary conditions given by the Method of Images. The electric potentials give the electric fields necessary to reach the steady state and thus gives us an estimate of the charge density and electric fields of a slowly developing storm. The model result of special importance to CAERENet is the electric field at ground level because CAERENet's electric field mills measure the electric field at the ground. Comparisons can be made between the models and the electric field mill data, which aids the interpretation of the latter. Figures 4.1-4.4 show the charge distribution, electric potential, electric field, and electric field at the ground output values when two long regions of current were used as the input. 

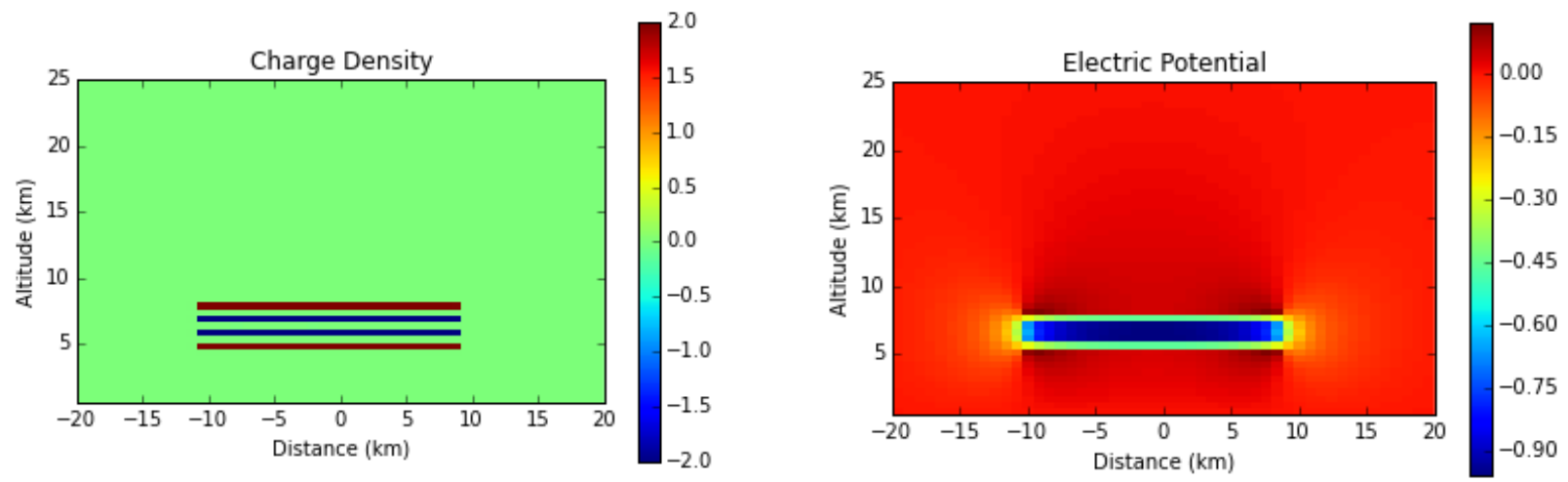

Figure 4-4.1-4.2: These figures show the charge distribution and the electric potential obtained as an output from the thunderstorm model when two long regions of current were used as inputs. This created a normal polarity tripole thunderstorm structure (positive bottom, overall negative middle, and positive top). The bars on the right of each panel indicate the positive and negative values for the colors in the figures. The actual values listed in the color bar are subject to rescaling.
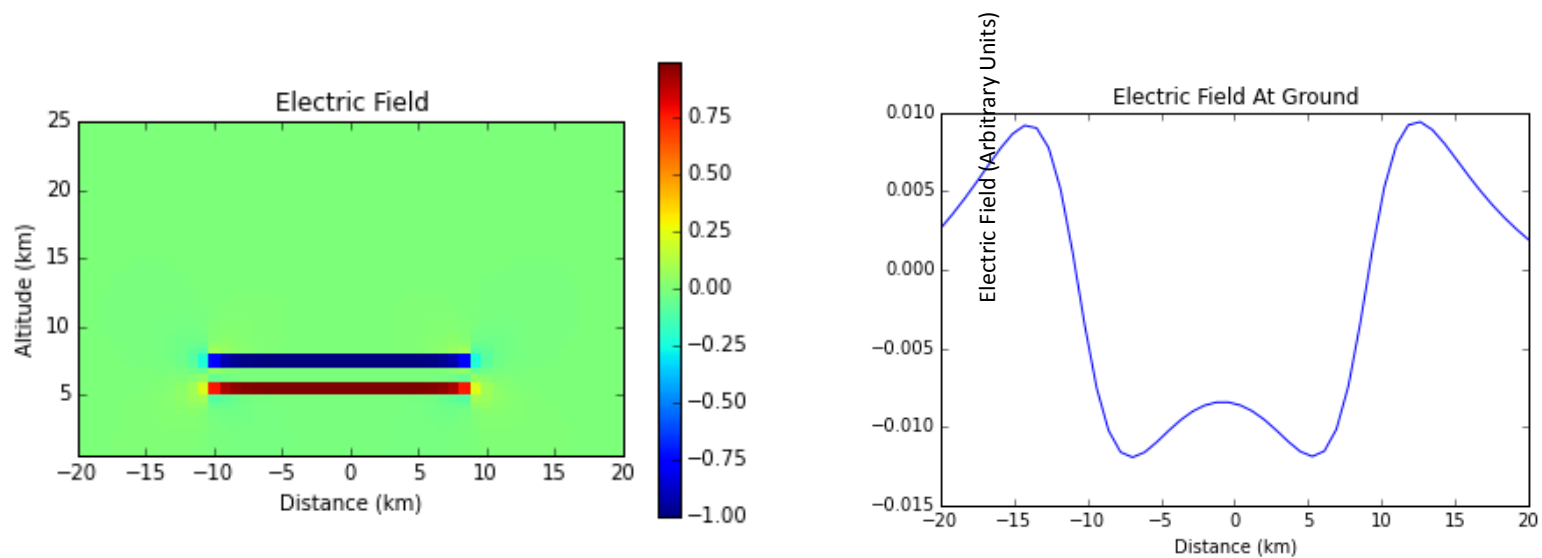

Figure 4.3-4.4: These figures show the electric field and the electric field at the ground obtained as an output from the thunderstorm model when two long regions of current were used as inputs. Figure 4.3 (left) indicates the electric field values throughout the entire model. The colors on the bar indicate the values for electric field, and are subject to rescaling similar to Figures 4.1 and 4.2. The blue values in the figure tend to be negative while the red values tend to be positive. Figure 4.4 (right) represents the electric field data in Figure 4.3 at the ground level. The values for electric field are in arbitrary units because some constant values were simplified in the model to allow the model to run at a reasonable speed.

\section{Results}

The goal of using the thunderstorm models was to be able to better understand the data from CAERENet's electric field mills. The models were analyzed for trends in the hopes that they would prove insightful when analyzing the electric field mill data. Some trends of interest were the reversal distance, or where the electric field mill data changed from positive to negative (the zero value), and the absolute minimum and maximum magnitude values. The parameters were changed inside of the model to see how these focus areas changed. One way the parameters were changed was by bringing a normal polarity tripole structure closer to the ground (the altitudes of the inputted current regions were changed), and the trends found are shown in Figures 5.1 and 5.2. As the storm structure moved closer to the ground, the reversal distance moved closer to the storm, and the absolute minimum and maximum magnitudes increased 

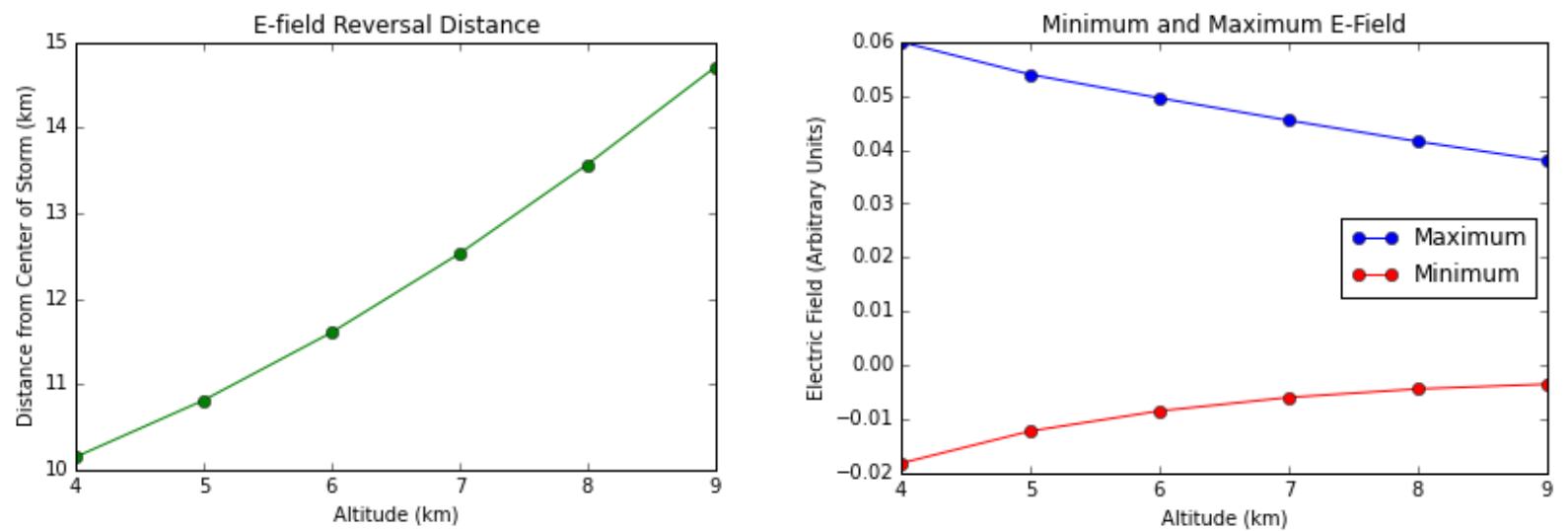

Figure 5-5.1 and 5.2: The trends found when a normal polarity tripole structure was moved closer to the ground are indicated here. The altitudes of the inputted current regions were changed for six simulations, and each simulation's reversal distance is indicated with a data point on each graph. Figure 5.1 (left) shows data for the reversal distance and Figure 5.2 (right) shows the data for the absolute minimum (red) and maximum (blue) magnitude values.

Besides moving a storm structure closer to the ground, the parameters were changed by varying the conductivity in the atmosphere and varying the number of current regions used to give extra regions of charge. The trends found using these parameters were more complicated and are yet to be fully understood. Therefore, they have not been included here. The CAERENet team hopes to collect experimental data with the electric field mills in the near future. The trends found with the Python models will continue to be evaluated in the future and be compared to electric field mill data, when the data is available, to better understand the data and the electrification processes at work inside of a thunderstorm.

\section{Concluding Remarks}

It is evident from this research project, as well as the literature available on the topic of thunderstorms, the electrification processes in thunderstorms are complicated and not yet well understood. Though studied in cloud chambers, there remains little evidence of the Relative Diffusional Growth Rate Theory in nature. In the future, the CAERENet team hopes to continue to evaluate trends from the Python models to understand the electric field mill data, which will hopefully shed light on the geometry and dynamics of charges within thunderstorms and thus help understand the electrification processes. This will hopefully indicate whether or not the Relative Diffusional Growth Rate Theory is playing a role in the electrification processes in naturally occurring thunderstorms.

\section{References}

Dash J, Mason B, Wettlaufer J, et. al. (2001). Theory of charge and mass transfer in ice-ice collisions. Journal of Geophysical Research 106, 20395-20402.

NOAA (2010). Glossary:G's. National Weather Service. Retrieved from http://www.srh.noaa.gov/jetstream/append/glossary_g.htm

Rust W., MacGorman D., Bruning E., et. al. (2005). Inverted-polarity electrical structures in thunderstorms in the Severe Thunderstorm Electrification and Precipitation Study (STEPS). Atmospheric Research, 76, 247-271. 
Saunders, C. (2008). Charge Separation Mechanisms in Clouds. Space Sci Rev 137, 335-353.

Stolzenburg M., Marshall T. (2008). Charge Structure and Dynamics in Thunderstorms. Space Sci Rev 137, 355-372. 